\title{
On the Theory of a Non-Periodic Quasilattice Associated with the Icosahedral Group
}

\author{
P. Kramer \\ Institut für Theoretische Physik der Universität Tübingen
}

Z. Naturforsch. 40 a, 775-788 (1985); received May 10, 1985

A quasilattice in the euclidean space $\mathbb{E}^{3}$ is generated by dualization of a hexagrid. The construction is based on a general theory of periodic and non-periodic space filling by projection from hypergrids. Global and local properties of the quasilattice are discussed, including the structure factor, the form and packing of the cells, and the point symmetry. The quasilattice is expected to give a description of quasicrystals without periodic order which have recently been found in experiments.

\section{Introduction}

In 1984 Shechtman, Blech, Gratias, and Cahn [1] reported on a metallic phase obtained by rapid cooling of an alloy formed from $\mathrm{Al}+14 \% \mathrm{Mn}$. The diffraction pattern of this phase shows icosahedral point symmetry. From this property the authors conclude that the phase cannot have periodic or translational order. From the sharpness of the diffraction pattern they infer that the metallic phase has long-range orientational order. These observations have been confirmed by other groups [2], [3].

A theory of periodic and non-periodic space fillings obtained by projection from hypergrids in $\mathbb{E}^{\mathrm{n}}$ was developed with Neri in [4]. In this theory, techniques due to de Bruijn [5] were generalized and connected with the representation theory of the translation and point groups in $\mathbb{E}^{\mathrm{n}}$. A particular non-periodic space filling of $\mathbb{E}^{3}$ is developed in [4] from the icosahedral group and its representations. In the present paper, this theory is presented and developed in more detail and with respect to the possible application to solid state physics which emerge in view of the experimental evidence on quasicrystals.

The paper deals with the following aspects of quasilattices: In Sect. 2 the hypercubic grid in $\mathbb{E}^{6}$ is analyzed with emphasis on its translational and point symmetry. The icosahedral group $A(5)$ is shown to be a subgroup of the point group of the hypercubic grid and yields, by reduction of its sixdimensional representation into two three-dimen-

Reprint requests to Prof. Dr. P. Kramer, Institut für Theoretische Physik, Universität Tübingen, Auf der Morgenstelle 14, D-7400 Tübingen. sional irreducible representations, a decomposition of $\mathbb{E}^{6}$ into two orthogonal subspaces $\mathbb{E}_{1}^{3}$ and $\mathbb{E}_{2}^{3}$. In Sect. 3 a hexagrid $Y_{1}$ in $\mathbb{E}^{3}$ is introduced which is interpreted as the projection of the hypercubic grid from $\mathbb{E}^{6}$ to $\mathbb{E}_{1}^{3}$. The hexagrid $Y_{1}$ is determined by six shift parameters and divides $\mathbb{E}^{3}$ into convex cells whose faces, edges and vertices are described. A subgrid description of the hexagrid $Y_{1}$ is given in terms of 20 normal grids.

A quasilattice $Z_{1}$ in another space $\mathbb{E}^{3}$ is constructed in Sect. 4 by dualization of the hexagrid. In the dualization, the dual objects between $Y_{1}$ and $Z_{1}$ are cells and dual vertices, faces and dual edges, edges and dual faces, vertices and dual cells.

In Sect. 5, a long-range approximation is formulated for the computation of the structure factor of the quasilattice $Z_{1}$. It is based on the subgrid description and yields an approximate but closed expression for the structure factor. This expression is compared to experiments.

In Sect. 6 the six shift parameter are studied. Fundamental regions are derived which determine the values of these parameters and yield hexagrids and quasilattices inequivalent under the symmetry groups involved. The analysis includes the possible point symmetry of the quasilattice. The local properties of the quasilattice are studied in Sects. 7 and 8 . It is shown that three types of composite cells and two types of elementary cells exist within the quasilattice. The formation of composite cells is linked to local symmetry breaking and possible jumps of faces in the quasilattice. To give more insight into the structure of the quasilattice, the packing of cells is analyzed in infinite two-dimensional layers. 


\section{The Cubic Grid in $\mathbb{E}^{6}$ and the Icosahedral Group A (5)}

In the euclidean space $\mathbb{E}^{6}$ we choose the orthonormal basis

$\left\{\boldsymbol{c}_{1}, \boldsymbol{c}_{2}, \ldots, \boldsymbol{c}_{6} \mid \boldsymbol{c}_{i} \cdot \boldsymbol{c}_{j}=\delta_{i j}, i, j=1,2, \ldots, 6\right\}$.

These vectors form the primitive basis vectors of the (hyper-)cubic lattice in $\mathbb{E}^{6}$. Let $\gamma$ be a fixed vector from $\mathbb{E}^{6}$ restricted by the conditions

$$
-1 / 2<\sqrt{2}\left(\gamma \cdot c_{i}\right) \leqq 1 / 2 .
$$

Define the system $Y^{i}$ of hyperplanes in $\mathbb{E}^{6}$ by

$Y^{i}=\left\{\boldsymbol{y} \mid \boldsymbol{y} \cdot \boldsymbol{c}_{i}=\frac{\sqrt{2}}{4} k_{i}-\boldsymbol{\gamma} \cdot \boldsymbol{c}_{i}, k_{i}= \pm 1, \pm 3, \ldots\right\}$.

These hyperplanes are parallel and for $\Delta k_{i}= \pm 2$ have the distance $1 / \sqrt{2}$. The hexagrid $Y$ of $\mathbb{E}^{6}$ is defined as the collection

$$
Y=Y^{1} Y^{2} \ldots Y^{6}
$$

of six systems of hyperplanes. The hexagrid $Y$ has as its point symmetry the hyperoctahedral group [6] in six dimensions which will be denoted as $\Omega(6)$. The elements of $\Omega(6)$ are the 6 ! permutations of the symmetric group $\mathrm{S}(6)$ and the six reflections with respect to the six axes. An element $f$ of $\Omega(6)$ is specified by giving six numbers for these reflections,

$$
\varepsilon_{i}(f)= \pm 1, \quad i=1,2, \ldots, 6,
$$

and a permutation

$$
f: i \rightarrow f(i), \quad i=1,2, \ldots, 6 .
$$

The representation of $\Omega(6)$ in $\mathbb{E}^{6}$ is then the $6 \times 6$ matrix with elements

$$
\tilde{D}_{i j}(f):=\varepsilon_{i}(f) \delta_{i, f(j)}, \quad i, j=1,2, \ldots, 6 .
$$

For short elements of $\Omega(6)$ are denoted in the form

$f:=\left|\begin{array}{cccccc}1 & 2 & 3 & 4 & 5 & 6 \\ \varepsilon_{1} f(1) & \varepsilon_{2} f(2) & \varepsilon_{3} f(3) & \varepsilon_{4} f(4) & \varepsilon_{5} f(5) & \varepsilon_{6} f(6)\end{array}\right|$.

As shown in [4] the icosahedral group A (5) can be embedded as a subgroup of $\Omega(6)$. The embedding is fixed by specification of the two generators of order 2 and 5 of $\mathrm{A}(5)$ respectively which are denoted by $g_{2}$ and $g_{5}$ and given in Table 1. In this table, the second description of the generators refers to permutations of 5 objects. These permutations
Table 1. The generators $g_{2}$ and $g_{5}$ of order 2 and 5 for the subgroup $\mathrm{A}(5)$ of $\Omega(6)$ in $\mathbb{E}^{6}$, the generator $g_{3}=g_{2} g_{5}$ of order 3 of $\mathrm{A}(5)$, and the generator $g_{4}$ of order 4 which yields an automorphism of A(5), in the notation of (2.8).

$\begin{aligned} g_{2} & :=\left|\begin{array}{rrrrrr}1 & 2 & 3 & 4 & 5 & 6 \\ 3 & 6 & 1 & -4 & -5 & 2\end{array}\right|, \text { permutation (bc) (de) } \\ g_{5} & :=\left|\begin{array}{rrrrrr}1 & 2 & 3 & 4 & 5 & 6 \\ 2 & 3 & 4 & 5 & 1 & 6\end{array}\right|, \text { permutation (abcde) } \\ g_{3} & =\left|\begin{array}{rrrrrr}1 & 2 & 3 & 4 & 5 & 6 \\ 6 & 1 & -4 & -5 & 3 & 2\end{array}\right|, \text { permutation (ace) } \\ g_{4} & :=\left|\begin{array}{rrrrrr}1 & 2 & 3 & 4 & 5 & 6 \\ 1 & 4 & 2 & 5 & 3 & -6\end{array}\right|\end{aligned}$

Table 2. Characters $\chi$ for irreducible representations of A (5). Class representatives are the identity element $e$, powers of the generators $g_{2}$ and $g_{5}$ and of $g_{3}=g_{2} g_{5}$. The number $\Phi$ is $\Phi=(1+\sqrt{5}) / 2$.

\begin{tabular}{lcc|c|rccc}
\hline $\begin{array}{l}\text { Class } \\
\text { represen- } \\
\text { tative }\end{array}$ & $\begin{array}{l}\text { Num- } \\
\text { ber of } \\
\text { elem. }\end{array}$ & \multicolumn{5}{l}{ Irreducible representation } \\
\cline { 4 - 7 } & 1 & 1 & 4 & 5 & 3 & 3 \\
\hline$e$ & 15 & 1 & 0 & 1 & -1 & -1 \\
$g_{2}$ & 20 & 1 & 1 & -1 & 0 & 0 \\
$g_{3}, g_{3}^{2}$ & 12 & 1 & -1 & 0 & $\Phi$ & $1-\Phi$ \\
$g_{5}, g_{5}^{4}$ & 12 & 1 & -1 & 0 & $1-\Phi$ & $\Phi$ \\
$g_{5}^{2}, g_{5}^{4}$ & 12 & 1 & &
\end{tabular}

are not meant to act on the space $\mathbb{E}^{6}$ but give an abstract form of A (5).

It was shown in [4] that the representation $\tilde{D}^{6}$ of $\Omega$ (6) under restriction to A (5) is reduced into irreducible representations $D^{\mathrm{i}}$ and $D^{\omega}$, each of dimension 3. The two representations may be identified by their characters given in Table 2 . The representation $D^{\mathrm{i}}$ consists of all proper rotations which form the symmetry operations of a regular dodecahedron or icosahedron. The reduction of the representation $\tilde{D}^{6}$ for $g \in \mathrm{A}(5)$ has the form

$$
\tilde{D}^{6}(g)=m^{-1}\left|\begin{array}{cc}
D^{\mathrm{i}}(g) & 0 \\
0 & D^{\omega}(g)
\end{array}\right| m,
$$

with the real orthogonal matrix $m$ given in Table 3 taken from [4].

Consider now the element $g_{4}$ of $\Omega(6)$ given in Table 1. This element is of order 4 and generates an outer automorphism of A (5)

$$
\sigma: \mathrm{A}(5) \rightarrow \mathrm{A}(5), \quad \sigma(g)=g_{4} g\left(g_{4}\right)^{-1} .
$$


Table 3. The matrix $m$ from [4] which reduces the representation according to (2.9). The abbreviations $\theta=\sqrt{1 / 5}$, $c=\cos (2 \pi / 5), s=\sin (2 \pi / 5), c^{\prime}=\cos (4 \pi / 5), s^{\prime}=\sin (4 \pi / 5)$ are used.

\begin{tabular}{lllllll}
\hline$m$ & 1 & 2 & 3 & 4 & 5 & 6 \\
\hline 1 & $\theta / \sqrt{2}$ & $\theta / \sqrt{2}$ & $\theta / \sqrt{2}$ & $\theta / \sqrt{2}$ & $\theta / \sqrt{2}$ & $1 / \sqrt{2}$ \\
2 & $2 \theta / \sqrt{2}$ & $2 \theta c / \sqrt{2}$ & $2 \theta c^{\prime} / \sqrt{2}$ & $2 \theta c^{\prime} / \sqrt{2}$ & $2 \theta c / \sqrt{2}$ & 0 \\
3 & 0 & $-2 \theta s / \sqrt{2}$ & $-2 \theta s^{\prime} / \sqrt{2}$ & $2 \theta s^{\prime} / \sqrt{2}$ & $2 \theta s / \sqrt{2}$ & 0 \\
4 & $-\theta / \sqrt{2}$ & $-\theta / \sqrt{2}$ & $-\theta / \sqrt{2}$ & $-\theta / \sqrt{2}$ & $-\theta / \sqrt{2}$ & $1 / \sqrt{2}$ \\
5 & $2 \theta / \sqrt{2}$ & $2 \theta c^{\prime} / \sqrt{2}$ & $2 \theta c / \sqrt{2}$ & $2 \theta c / \sqrt{2}$ & $2 \theta c^{\prime} / \sqrt{2}$ & 0 \\
6 & 0 & $-2 \theta s^{\prime} / \sqrt{2}$ & $2 \theta s / \sqrt{2}$ & $-2 \theta s / \sqrt{2}$ & $2 \theta s^{\prime} / \sqrt{2}$ & 0 \\
\hline
\end{tabular}

An explicit computation of the representation yields

$$
\begin{aligned}
& \tilde{D}^{6}\left(g_{4}\right)=m^{-1}\left|\begin{array}{cc}
0 & d^{1} \\
d^{2} & 0
\end{array}\right| m, \\
& d^{1}=\left|\begin{array}{rrr}
-1 & 0 & 0 \\
0 & 1 & 0 \\
0 & 0 & -1
\end{array}\right|, \quad d^{2}=\left|\begin{array}{rrr}
-1 & 0 & 0 \\
0 & 1 & 0 \\
0 & 0 & 1
\end{array}\right| .
\end{aligned}
$$

Denoting by $D^{6}$ the representation of $\Omega(6)$ given by

$$
D^{6}=m \tilde{D}^{6} m^{-1}
$$

the automorphism property yields

$$
\tilde{D}^{6}(\sigma(g))=\tilde{D}^{6}\left(g_{4}\right) \tilde{D}^{6}(g) \tilde{D}^{6}\left(g_{4}^{-1}\right)
$$

and

$$
D^{6}(\sigma(g))=D^{6}\left(g_{4}\right) D^{6}(g) D^{6}\left(g_{4}^{-1}\right) .
$$

Using the explicit form (2.9) of $D(g)$ and the matrices $d^{1}$ and $d^{2}$ of (2.12) one finds the relations

$$
\begin{aligned}
& D^{\mathrm{i}}(\sigma(g))=d^{1} D^{\omega}(g)\left(d^{1}\right)^{-1}, \\
& D^{\omega}(\sigma(g))=d^{2} D^{\mathrm{i}}(g)\left(d^{2}\right)^{-1} .
\end{aligned}
$$

These equations show that the representations $D^{\mathrm{i}}$ and $D^{\omega}$ are linked to each other by the automorphism $\sigma$. If $D^{\mathrm{i}}(\mathrm{g})$ runs over the rotations which are symmetry operations of the dodecahedron, then $D_{2}^{\omega}(\sigma(g))$ runs over the same rotations transformed by the matrix $d^{2}$. This property will be used in Sections 6 .

The matrix $m$ provides an explicit orthogonal decomposition of $\mathbb{E}^{6}$,

$$
\mathbb{E}^{6}=\mathbb{E}_{1}^{3}+\mathbb{E}_{2}^{3},
$$

where the indices 1 and 2 denote the representation spaces for $D^{\mathrm{i}}$ and $D^{\omega}$ respectively. A basis for the two spaces is provided by the vectors

$$
\mathbb{E}_{1}^{3}: \boldsymbol{d}_{i}=\sum_{j=1}^{6} m_{i j} \boldsymbol{c}_{j}, \quad i=1,2,3,
$$

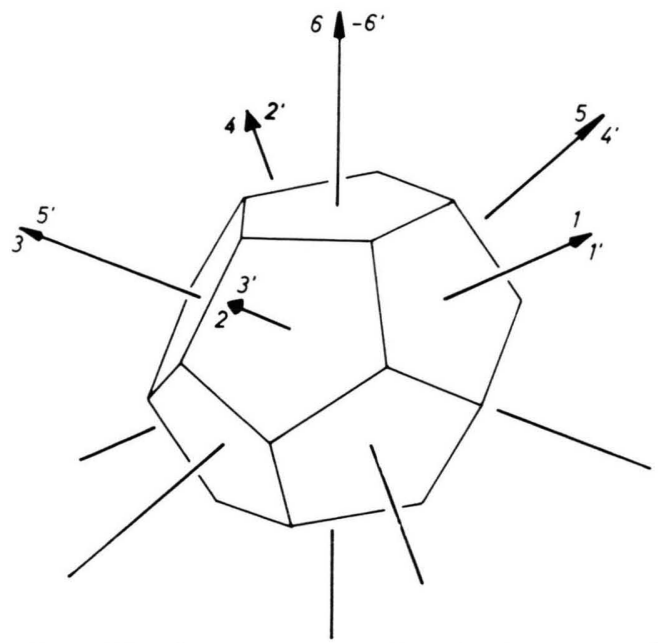

Fig. 1. The six vectors $\boldsymbol{e}_{i 1}$ and $\boldsymbol{e}_{i 2}$ in the spaces $\mathbb{E}_{1}^{3}$ and $\mathbb{E}_{2}^{3}$ for $i=1,2, \ldots, 6$ are marked by the unprimed and primed numbers respectively. They are orthogonal to six pairs of parallel faces of the regular dodecahedron.

Table 4. The six vectors $\boldsymbol{e}_{i 1}$ and $\boldsymbol{e}_{i 2}$ in the spaces $\mathbb{E}_{1}^{3}$ and $\mathbb{E}_{2}^{3}$ written as column vectors. The notation of Table 3 is applied.

\begin{tabular}{lllllll}
\hline$i$ & 1 & 2 & 3 & 4 & 5 & 6 \\
\hline $\boldsymbol{e}_{j, i 1}$ & $\theta$ & $\theta$ & $\theta$ & $\theta$ & $\theta$ & 1 \\
& $2 \theta$ & $2 \theta c$ & $2 \theta c^{\prime}$ & $2 \theta c^{\prime}$ & $2 \theta c$ & 0 \\
& 0 & $-2 \theta s$ & $-2 \theta s^{\prime}$ & $2 \theta s^{\prime}$ & $2 \theta s$ & 0 \\
$\boldsymbol{e}_{j, i 2}$ & $-\theta$ & $-\theta$ & $-\theta$ & $-\theta$ & $-\theta$ & 1 \\
& $2 \theta$ & $2 \theta c^{\prime}$ & $2 \theta c$ & $2 \theta c$ & $2 \theta c^{\prime}$ & 0 \\
& 0 & $-2 \theta s^{\prime}$ & $2 \theta s$ & $-2 \theta s$ & $2 \theta s^{\prime}$ & 0 \\
\hline
\end{tabular}

$$
\mathbb{E}_{2}^{3}: \boldsymbol{d}_{k}=\sum_{j=1}^{6} m_{k j} \boldsymbol{c}_{j}, \quad k=4,5,6 .
$$

Conversely one may take the vectors $\boldsymbol{d}_{i}$ as standard unit vectors in the two spaces. Then the projection of the vectors $\boldsymbol{c}_{i}$ into the two spaces are given by $\boldsymbol{c}_{i 1}, \boldsymbol{c}_{i 2}$ where

$$
\begin{aligned}
c_{j, i 1} & =(1 / 2)^{1 / 2} e_{j, i 1}=m_{j i}, \\
j & =1,2,3, \quad i=1,2, \ldots, 6, \\
c_{j, i 2} & =(1 / 2)^{1 / 2} e_{j, i 2}=m_{j+3, i}, \\
& \quad j=1,2,3, \quad i=1,2, \ldots, 6 .
\end{aligned}
$$

The index $i$ denotes the index of the basis vector and the index $j$ its component. The vectors $\boldsymbol{e}_{i 1}$ and $\boldsymbol{e}_{i 2}, i=1,2, \ldots, 6$ are found to be orthogonal to six different faces of regular dodecahedra in $\mathbb{E}_{1}^{3}$ and $\mathbb{E}_{2}^{3}$ respectively, compare Figure 1. They have been normalized to the length one and are given in Table 4. 
To summarize we have described in this section a hypercubic grid in $\mathbb{E}^{6}$ and its point symmetry group $\Omega$ (6). The subgroup A (5) yields a decomposition of the space into subspaces $\mathbb{E}_{1}^{3}$ and $\mathbb{E}_{2}^{3}$ which carry irreducible representation $D^{\mathrm{i}}$ and $D^{\omega}$ respectively. These representations are linked by an outer automorphism of $\mathrm{A}(5)$. Finally we mention that in [4] the analysis is implemented in a space $\mathbb{E}^{12}$ which then is projected into two subspaces $\mathbb{E}_{1}^{6}$ and $\mathbb{E}_{2}^{6}$. This first projection is used in [4] to give an alternating orientation to the hypercubic lattice in $\mathbb{E}^{6}$ analyzed in the present paper.

\section{The Hexagrid $Y_{1}$ in $\mathbb{E}^{3}$}

Consider in $\mathbb{E}^{3}$ a regular dodecahedron and six vectors $\boldsymbol{e}_{i}, i=1,2, \ldots, 6$ of unit length and orthogonal to six different faces respectively. Define six real shift parameters $\gamma_{i}$ restricted by

$$
-\frac{1}{2}<\gamma_{i} \leqq \frac{1}{2}, \quad i=1,2, \ldots, 6
$$

and introduce the system of equidistant consecutive parallel planes

$Y^{i}=\left\{\boldsymbol{y} \in \mathbb{E}^{3} \mid \boldsymbol{y} \cdot \boldsymbol{e}_{i}=\frac{1}{2} k_{i}-\gamma_{i}, k_{i}= \pm 1, \pm 3, \ldots\right\}$.

A hexagrid $Y_{1}$ in $\mathbb{E}^{3}$ is a collection of six systems $Y^{i}$,

$$
Y_{1}=Y^{1}, Y^{2}, \ldots, Y^{6}
$$

Without loss of generality, the hexagrid $Y_{1}$ of $\mathbb{E}^{3}$ may be obtained by projection from $\mathbb{E}^{6}$. The six vectors $\boldsymbol{e}_{i}$ may be taken as the vector $\boldsymbol{e}_{i 1}$ discussed in Sect. 2 and given in Table 4. The shift parameters $\gamma_{i}$ can be interpreted as

$$
\gamma_{i}=\sqrt{2}\left(\gamma \cdot \boldsymbol{c}_{i}\right)=\left(\gamma \cdot \boldsymbol{e}_{i 1}+\gamma \cdot \boldsymbol{e}_{i 2}\right),
$$

where

$$
c_{i}=\sqrt{1 / 2}\left(e_{i 1}+e_{i 2}\right)
$$

is the projection of the basis for the cubic lattice in $\mathbb{E}^{6}$ into the subspaces $\mathbb{E}_{1}^{3}$ and $\mathbb{E}_{2}^{3}$. The space $\mathbb{E}^{3}=\mathbb{E}_{1}^{3}$ is the space where the hexagrid $Y_{1}$ is defined. The space $\mathbb{E}^{6}$ will be used for the analysis of the shift parameters $\gamma_{i}$ in Section 6.

The system $Y^{i}$ divides $\mathbb{E}^{3}$ into zones whose points $y$ obey

$$
\frac{1}{2}\left(k_{i}-2\right)<\left(\boldsymbol{y} \cdot \boldsymbol{e}_{i 1}\right)+\gamma_{i} \leqq \frac{1}{2} k_{i} .
$$

Define for real numbers $\alpha$ the function

$$
\Gamma^{-}: \alpha \rightarrow \alpha^{-}
$$

as the smallest integer number larger or equal to $\alpha$. Then the zone index $k_{i}$ for a given point $\boldsymbol{y} \in \mathbb{E}^{3}$ is obtained as

$$
k_{i}(\boldsymbol{y})=2\left\lceil\left(\boldsymbol{y} \cdot \boldsymbol{e}_{i 1}+\gamma_{i}-\frac{1}{2}\right)\right\rceil+1 .
$$

Extending this analysis to all six systems $Y^{j}$, $j=1,2, \ldots, 6$, the six systems of zones subdivide $\mathbb{E}^{3}$ into cells. For short we shall refer to this system of cells as the hexagrid $Y_{1}$. Any point $\boldsymbol{y} \in \mathbb{E}^{3}$ belongs to one and only one cell which may be found from the six functions $k_{i}(y), i=1,2, \ldots, 6$ according to (3.8). These functions are constant on a fixed cell and we call the set of functions

$\boldsymbol{y} \rightarrow\left\{k_{1}(\boldsymbol{y}), k_{2}(\boldsymbol{y}), k_{3}(\boldsymbol{y}), k_{4}(\boldsymbol{y}), k_{5}(\boldsymbol{y}), k_{6}(\boldsymbol{y})\right\}$

the index system of the cell.

Any face of a cell of the hexagrid $Y_{1}$ is part of a plane associated with some fixed index $k_{j}$. When crossing the face to the next cell with the same face, the index systems are related in the single index $j$ according to

$$
k_{j}^{\prime}=k_{j}+\delta_{j}, \quad \delta_{j}=0,2 .
$$

Any edge of a cell of the hexagrid $Y_{1}$ is part of the intersection of two planes, say $j$ and $l$, and is common to four cells. The index systems of these four cells differ only in the components $j$ and $l$. Choosing the cell with the lowest values of $k_{j}$ and $k_{l}$, the indices of the four cells with a common edge are related by

$$
k_{\alpha}^{\prime}=\left\{\begin{array}{ccc}
k_{\alpha}+\delta_{j}+\delta_{l} & \text { if } & \alpha \in(j, l), \\
k_{\alpha} & \text { if } & \alpha \notin(j, l) .
\end{array}\right.
$$

A vertex of the hexagrid $Y_{1}$ is the intersection of $p$ planes, $p=3,4,5,6$. We call the vertex regular if $p=3$. A regular vertex is common to eight cells of $Y$ and associated to three planes, say $j, l$, and $i$. Choosing the cell with the lowest components $k_{j}, k_{l}$, $k_{i}$ for reference, the indices of the eight cells with a common regular vertex are related by

$$
k_{\alpha}^{\prime}=\left\{\begin{array}{ccc}
k_{\alpha}+\delta_{j}+\delta_{l}+\delta_{i} & \text { if } & \alpha \in(j, l, i), \\
k_{\alpha} & \text { if } & \alpha \notin(j, l, i) .
\end{array}\right.
$$

We now give a subgrid description of the hexagrid $Y_{1}$ for the case that all vertices are regular. In this case, all vertices of $Y_{1}$ can be determined by the construction of 20 subgrids formed from $\left(\begin{array}{l}6 \\ 3\end{array}\right)=20$ triples of vectors from the set

$$
\boldsymbol{e}_{i}, \quad i=1,2, \ldots, 6 .
$$


Table 5. The triples of vectors $(i j l)=\left(\tilde{\boldsymbol{e}}_{i} \tilde{\boldsymbol{e}}_{j} \tilde{\boldsymbol{e}}_{l}\right)$ for $v=1,2$, $\ldots, 20$ in the short-hand notation $\boldsymbol{e}_{i} \rightarrow i,-\boldsymbol{e}_{i} \rightarrow \bar{i}$.

\begin{tabular}{llllllllll}
\hline 1 & 2 & 3 & 4 & 5 & 6 & 7 & 8 & 9 & 10
\end{tabular}

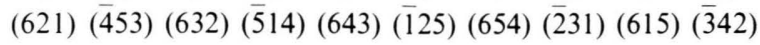

$\begin{array}{llllllllll}11 & 12 & 13 & 14 & 15 & 16 & 17 & 18 & 19 & 20\end{array}$ $(2 \overline{4} 1)(\overline{536})(3 \overline{5} 2)(\overline{14} 6)(4 \overline{1} 3)(\overline{25} 6)(5 \overline{2} 4)(\overline{31} 6)(1 \overline{3} 5)(\overline{42} 6)$

Table 6. The scalar products $\left(\boldsymbol{e}_{\alpha} \cdot \boldsymbol{e}_{\gamma}\right)=\left(\boldsymbol{e}_{\gamma} \cdot \boldsymbol{e}_{\alpha}\right)$.

\begin{tabular}{l|llrrrr}
\hline & 1 & 2 & 3 & 4 & 5 & 6 \\
\hline 1 & 1 & $\theta$ & $-\theta$ & $-\theta$ & $\theta$ & $\theta$ \\
2 & & 1 & $\theta$ & $-\theta$ & $-\theta$ & $\theta$ \\
3 & & & 1 & $\theta$ & $-\theta$ & $\theta$ \\
4 & & & & 1 & $\theta$ & $\theta$ \\
5 & & & & & 1 & $\theta$ \\
6 & & & & & & 1 \\
\hline
\end{tabular}

A systematic choice of these triples is given in Table 5. We denote a fixed triple $v$ as

$$
v \rightarrow\left(\tilde{\boldsymbol{e}_{i}} \tilde{\boldsymbol{e}_{j}} \tilde{\boldsymbol{e}_{l}}\right),
$$

where the replacement $\tilde{\boldsymbol{e}}_{i 1}=\tilde{\boldsymbol{e}}_{i}$ by $\tilde{\boldsymbol{e}}_{i}$ includes the necessary changes of sign according to Table 5. The triples have been arranged to yield the metrical subgrid properties obtained from Table 6 ,

$$
\begin{aligned}
& v=\text { odd: }\left(g_{i j}(v)\right)=\left(\tilde{\boldsymbol{e}}_{i} \cdot \tilde{\boldsymbol{e}}_{j}\right)=\left|\begin{array}{lll}
1 & \theta & \theta \\
\theta & 1 & \theta \\
\theta & \theta & 1
\end{array}\right|, \\
& v=\text { even: }\left(g_{i j}(v)\right)=\left(\tilde{\boldsymbol{e}}_{i} \cdot \tilde{\boldsymbol{e}}_{j}\right)=\left|\begin{array}{rrr}
1 & -\theta & -\theta \\
-\theta & 1 & -\theta \\
-\theta & -\theta & 1
\end{array}\right|, \\
& \theta=\sqrt{1 / 5}
\end{aligned}
$$

For a fixed triple $v$ we define the dual basis by

$$
\begin{aligned}
& \tilde{\boldsymbol{e}}^{j}(v): \tilde{\boldsymbol{e}}^{j}(v) \cdot \tilde{\boldsymbol{e}}_{i}(v)=\delta_{j i}, \\
& \tilde{\boldsymbol{e}}_{i}(v)=\sum_{l} g_{i l}(v) \tilde{\boldsymbol{e}}^{l}(v)
\end{aligned}
$$

and note that the matrix inverse to $\left(g_{i j}\right)$ is

$$
\left(g^{i j}(v)\right): g^{i j}(v)=\tilde{\boldsymbol{e}}^{i}(v) \cdot \tilde{\boldsymbol{e}}^{j}(v) .
$$

For a fixed triple $v$, we denote by $\beta$ the indices not contained in the set $\left(\tilde{\boldsymbol{e}}_{i}, \tilde{\boldsymbol{e}}_{j}, \tilde{\boldsymbol{e}}_{l}\right)$. For the vectors $\boldsymbol{e}_{\beta}$ one easily derives the expressions

$$
\begin{aligned}
& \boldsymbol{e}_{\beta}=\sum_{l} f_{\beta l}(v) \boldsymbol{e}_{l}(v), \\
& f_{\beta l}(v)=\sum_{j}\left(\boldsymbol{e}_{\beta} \cdot \tilde{\boldsymbol{e}}_{j}(v)\right) g^{j l}(v) .
\end{aligned}
$$

For a fixed point $x \in \mathbb{E}^{3}$ one finds then

$$
\boldsymbol{x} \cdot \boldsymbol{e}_{\beta}=\sum_{j}\left(\boldsymbol{x} \cdot \tilde{\boldsymbol{e}}_{j}\right) f_{\beta j}(v) .
$$

The sums in $(3.20)-(3.22)$ run only over the three indices of the triple $v$.

In the present section we described a hexagrid $Y_{1}$ in $\mathbb{E}^{3}$ determined by 6 shift parameters $\gamma_{i}$, $i=1,2, \ldots, 6$. More details of this hexagrid will be discussed in Sections 5-8.

\section{The Quasilattice $Z_{1}$ in $\mathbb{E}^{3}$}

To the hexagrid $Y_{1}$ in $\mathbb{E}^{3}$ we associate a dual quasilattice and space filling $Z_{1}$ in a new space $\mathbb{E}^{3}$. The dual cells, faces, edges and vertices of $Z_{1}$ correspond to the vertices, edges, faces and cells of the hexagrid $Y_{1}$ respectively.

Given a cell of $Y_{1}$ with the index system $\left(k_{1}, k_{2}\right.$, $\left.k_{3}, k_{4}, k_{5}, k_{6}\right)$, the corresponding vertex of $Z_{1}$ is defined with the position vector

$$
v=\frac{1}{2} \sum_{\alpha=1}^{6} k_{\alpha} e_{\alpha 1}
$$

where the vectors $\boldsymbol{e}_{i 1}, i=1,2, \ldots, 6$ are now considered in a new space $\mathbb{E}^{3}$. Given two cells with a common face and two points $\boldsymbol{y}$ and $\boldsymbol{y}^{\prime}$ respectively, we have in the dual space $\mathbb{E}^{3}$ from $(3.10,4.1)$ the relation

$$
v\left(y^{\prime}\right)=v(y)+e_{j} .
$$

We connect all vertices of $Z_{1}$ which are dual to pairs of cells of $Y_{1}$ with a common face to obtain a directed graph $Z_{1}$.

Consider the part of $Z_{1}$ dual to four cells of $Y_{1}$ with a common edge according to (3.11). The four dual vertices of $Z_{1}$ have the position vectors

$$
\boldsymbol{v}=\frac{1}{2} \sum_{\alpha=1}^{6} k_{\alpha} \boldsymbol{e}_{\alpha 1}+\frac{1}{2}\left(\delta_{j} \boldsymbol{e}_{j 1}+\delta_{l} \boldsymbol{e}_{l 1}\right)
$$

The corresponding part of the directed graph $Z_{1}$ is a rhombus whose vectors $\boldsymbol{e}_{j}, \boldsymbol{e}_{l}$ have the angle $\varphi$,

$$
\cos \varphi=\boldsymbol{e}_{j} \cdot \boldsymbol{e}_{l}= \pm \theta,
$$

compare Table 6 . We convert this rhombus into a face of the dual object $Z_{1}$. All these faces have the same shape.

Next we consider the part of $Z_{1}$ dual to eight cells of $Y_{1}$ (which for the moment we assume to be regular) sharing a vertex of $Y_{1}$. The eight dual vertices of $Z_{1}$ have from $(3.12,4.1)$ the positions

$$
\boldsymbol{v}=\frac{1}{2} \sum_{\alpha=1}^{6} k_{\alpha} \boldsymbol{e}_{\alpha 1}+\frac{1}{2}\left(\delta_{j} \boldsymbol{e}_{j 1}+\delta_{l} \boldsymbol{e}_{l 1}+\delta_{i} \boldsymbol{e}_{i 1}\right)
$$



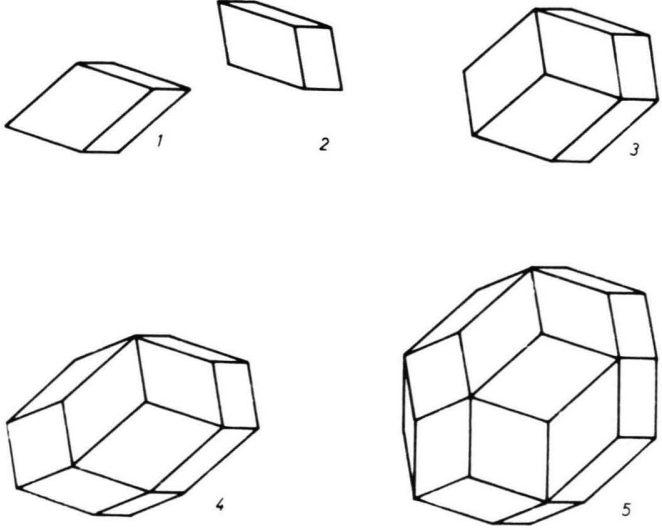

Fig. 2. The cells of the quasilattice $Z_{1}$ in $\mathbb{E}^{3}$ are zonohedra whose faces have a single rhombus shape. The cells 1 and 2 are the two rhombic hexahedra $h, h^{\prime}$ discussed in Section 4 , the cells 3,4 and 5 are the rhombic dodecahedron $d$, ikosahedron $i$ and triakontahedron $t$ discussed in Section 7.

These vertices from a rhombohedron of $Z_{1}$ which has twelve rhombus faces of the same type. There are two types of rhombohedra which correspond to the two types of triples of vectors with metrical properties described in (3.15) and (3.16) respectively for $v=$ odd and $v=$ even. Each type of rhombohedral cell of $Z_{1}$ appears in 10 possible orientations according to the triples given in Table 5. The rhombohedral cells are shown in Fig. 2, we refer to them as the thick and thin cell. These cells were considered before by Mackay [7]. We summarize the description of the dual quasilattice $Z_{1}$. The vertices of $Z_{1}$ are obtained as dual objects of the cells of $Y_{1}$ and have the positions $v$ specified in (4.1). Pairs of vertices of $Z_{1}$ are connected by vectors from the set $\boldsymbol{e}_{i}, i=1,2, \ldots, 6$ to form the edges of a directed graph. This directed graph consists of plane loops of a single rhombus shape connecting four neighbouring vertices respectively. The rhombus loops form a system of plane rhombus faces of $Z_{1}$. For a regular hexagrid $Y_{1}$, sets of twelve rhombus faces form rhombohedral cells of two types. Each type appears in ten possible orientations. The collection of all these cells forms a space filling $Z_{1}$ of $\mathbb{E}^{3}$. Thus the object $Z_{1}$ dual to the hexagrid $Y_{1}$ determines a quasilattice, a directed graph and an infinite space filling. Different quasilattices are dinstinguished by the values of the shift parameters $\gamma_{i}, i=1,2, \ldots, 6$. This dependence will be discussed in more detail in Section 6 .

\section{Long-Range Properties of the Quasilattice and the Structure Factor}

We consider a regular hexagrid $Y_{1}$ of $\mathbb{E}^{3}$ and make use of the subgrid description developed in Section 3. For a fixed number $v$ and a corresponding triple of vectors we take a cell which contains the vertex

$$
\boldsymbol{x}: \boldsymbol{x} \cdot \tilde{\boldsymbol{e}}_{j}=\frac{1}{2} k_{j}-\tilde{\gamma}_{j}, \quad j \text { from the triple } v .
$$

The corresponding vertex of $Z_{1}$ we denote by

$$
v\left(k_{i}, v\right)=\frac{1}{2} \sum_{j} k_{j} \tilde{\boldsymbol{e}}_{j}(v)+\frac{1}{2} \sum_{\beta} k_{\beta} \boldsymbol{e}_{\beta} .
$$

The index $j$ runs over the triple $v$ and the index $\beta$ over the indices not contained in this triple, and the values $k_{\beta}$ are given by

$$
\frac{1}{2}\left(k_{\beta}-2\right)<\boldsymbol{x} \cdot \boldsymbol{e}_{\beta}+\gamma_{\beta} \leqq \frac{1}{2} k_{\beta} .
$$

We introduce the numbers

$$
-\mu_{\beta}(\boldsymbol{x})=\left(\boldsymbol{x} \cdot \boldsymbol{e}_{\beta}\right)+\gamma_{\beta}-\frac{1}{2} k_{\beta}, 0 \leqq \mu_{\beta}(\boldsymbol{x})<1
$$

and use them to rewrite $v\left(k_{i}, v\right)$ in the form

$$
v\left(k_{i}, v\right)=\boldsymbol{v}_{a}\left(k_{i}, v\right)+v_{b}\left(k_{\beta}, v\right),
$$

where

$$
\begin{aligned}
& \boldsymbol{v}_{a}\left(k_{i}, v\right)= \frac{1}{2} \sum_{j} k_{j} \tilde{\boldsymbol{e}}_{i}(v) \\
&+\sum_{\beta}\left(\left(\boldsymbol{x} \cdot \boldsymbol{e}_{\beta}\right)+\sum_{j} \tilde{\gamma}_{j}\left(\tilde{\boldsymbol{e}}^{j} \cdot \boldsymbol{e}_{\beta}\right)\right) \boldsymbol{e}_{\beta}, \\
& \boldsymbol{v}_{b}\left(k_{i}, v\right)=\sum_{\beta}\left(\gamma_{\beta}+\mu_{\beta}(\boldsymbol{x})-\sum_{j} \tilde{\gamma}_{j}\left(\tilde{\boldsymbol{e}}^{j} \cdot \boldsymbol{e}_{\beta}\right)\right) \boldsymbol{e}_{\beta} .
\end{aligned}
$$

For the vector $v_{a}\left(k_{i}, v\right)$ we now claim the key result

$$
\begin{aligned}
& \boldsymbol{v}_{a}\left(k_{i}, v\right)=\frac{1}{2} \sum_{j} k_{j} \boldsymbol{a}_{j}, \\
& \boldsymbol{a}_{j}=2 \tilde{\boldsymbol{e}}^{j}(v) .
\end{aligned}
$$

For the proof we equate (5.6) and (5.8) to obtain

$$
\boldsymbol{a}_{j}=\sum_{l} Q_{j l} \tilde{\boldsymbol{e}}_{l}(v),
$$

where

$$
\begin{aligned}
Q_{j l}: & =\delta_{j l}+\sum_{i, m} \sum_{\beta} g^{j i}\left(\tilde{\boldsymbol{e}}_{i} \cdot \boldsymbol{e}_{\beta}\right)\left(\boldsymbol{e}_{\beta} \cdot \tilde{\boldsymbol{e}}_{m}\right) g^{m l} \\
& =\sum_{i, m} g^{j i} V_{i m} g^{m l}
\end{aligned}
$$

and

$$
V_{i m}:=\sum_{x=1}^{6}\left(\tilde{\boldsymbol{e}}_{i} \cdot \boldsymbol{e}_{x}\right)\left(\boldsymbol{e}_{x} \cdot \tilde{\boldsymbol{e}}_{m}\right) .
$$


An explicit computation for $v=1,2$, using Table 6, yields in both cases

$$
V_{i m}=V_{i m}(v)=2 g_{i m}(v)
$$

which then is a general result for all $v$. Now we get

$$
Q_{j l}=Q_{j l}(v)=2 g^{j l}(v)
$$

which completes the proof of (5.9).

Now we formulate a long-range approximation for the hexagrid $Y_{1}$ by restricting the attention to the vertices $\boldsymbol{x}$ of the subgrid $v$ which obey

$$
\left(\boldsymbol{x} \cdot \boldsymbol{e}_{j}(v)\right) \gg 1, \quad\left(\boldsymbol{x} \cdot \boldsymbol{e}_{\beta}\right) \gg 1 .
$$

The vertices $x$ which do not obey these inequalities are close to six fixed planes in the hexagrid and we argue that for global properties they can be neglected in lowest order.

In the long-range approximation, the quantities $\gamma_{\beta}+\mu_{\beta}(\boldsymbol{x})$ may be omitted and one obtains

$$
\boldsymbol{v}\left(k_{i}, v\right)_{\mathrm{app}}=\boldsymbol{v}_{a}\left(k_{i}, v\right)=\frac{1}{2} \sum_{j} k_{j} \boldsymbol{a}_{j} .
$$

The interpretation of the long-range approximation from (5.16) is that the vertices of $Z_{1}$ dual to the subgrid $v$ in this approximation occupy the intersection points of an ordinary grid formed from three sets of parallel planes orthogonal to the three vectors from the triple $v$. The distance of consecutive parallel planes is 2 in this case. The same reasoning applies to all vertices of $Z_{1}$ obtained from subgrids of the hexagrid $Y_{1}$.

Now we consider the amplitude function [8] of the quasilattice $Z_{1}$ given by

$$
A(\Delta \boldsymbol{k})=\sum_{\boldsymbol{v} \in Z_{1}} \exp i(\Delta \boldsymbol{k} \cdot \boldsymbol{v}), \quad \Delta \boldsymbol{k}=\boldsymbol{k}-\boldsymbol{k}^{\prime},
$$

where $\boldsymbol{k}$ and $\boldsymbol{k}^{\prime}$ are the wave vector of the ingoing and the outgoing diffracted beam respectively.

The amplitude function $A$ can be evaluated in closed form in the long-range approximation. For this purpose we rewrite $\boldsymbol{v}_{a}\left(k_{i}, v\right)$ in the form

$$
\begin{aligned}
& \boldsymbol{v}_{a}\left(k_{i}, v\right)=\frac{1}{2} \sum_{j}\left(k_{j}-1\right) \boldsymbol{a}_{j}(v)+\boldsymbol{w}(v), \\
& \boldsymbol{w}(v)=\frac{1}{2} \sum_{j} \boldsymbol{a}_{j}(v),
\end{aligned}
$$

so that $\frac{1}{2}\left(k_{j}-1\right)$ takes the values $0, \pm 1, \pm 2, \ldots$ We split the sum in (5.17) into 20 sums corresponding to the values of $v$ and perform the summation over the subgrids according to standard lattice theory [8] to obtain

$$
\begin{aligned}
& A(\Delta \boldsymbol{k})_{\mathrm{app}} \\
& =\sum_{v=1}^{20}(2 \pi)^{3} \frac{N(v)}{\Omega} \sum_{\boldsymbol{K}(v)} \delta(\Delta \boldsymbol{k}, \boldsymbol{K}(v)) \exp i(\Delta \boldsymbol{k} \cdot \boldsymbol{w}(v)) .
\end{aligned}
$$

Here $N(v)$ is the number of sublattice cells in the volume $\Omega$, and $\boldsymbol{K}(v)$ runs over the reciprocal sublattice for the index $v$. Then we have

$$
\boldsymbol{K}(v)=2 \pi \sum_{l} h_{l} \boldsymbol{b}_{l}(v), \quad h_{l}=0, \pm 1, \pm 2, \ldots,
$$

where $\boldsymbol{b}_{l}(v)$ are the basis vectors reciprocal to $\boldsymbol{a}_{l}(v)$. From (5.9) one finds

$$
\boldsymbol{b}_{l}(v)=\frac{1}{2} \tilde{\boldsymbol{e}}_{l}(v) \text {. }
$$

The structure factor is given by

$$
S(\Delta \boldsymbol{k})=|A(\Delta \boldsymbol{k})|^{2}
$$

and in long-range approximation yields

$$
\begin{aligned}
S(\Delta \boldsymbol{k})_{\mathrm{app}}= & \sum_{v=1}^{20} \sum_{v^{\prime}=1}^{20}(2 \pi)^{6} \frac{N(v) N\left(v^{\prime}\right)}{\Omega^{2}} \\
& \cdot\left(\sum_{\boldsymbol{K}(v)} \sum_{\boldsymbol{K}\left(v^{\prime}\right)} \delta(\Delta \boldsymbol{k}, \boldsymbol{K}(v)) \delta\left(\Delta \boldsymbol{k}, \boldsymbol{K}\left(v^{\prime}\right)\right)\right. \\
& \cdot \exp i\left(\Delta \boldsymbol{k} \cdot\left(\boldsymbol{w}(v)-\boldsymbol{w}\left(v^{\prime}\right)\right)\right) .
\end{aligned}
$$

The part $v=v^{\prime}$ of the structure factor yields the sum of the 20 structure factors from the 20 sublattices. The terms $v \neq v^{\prime}$ are interference contributions from different sublattices.

In Fig. 3 we show a partial result of the expression (5.24). All points $\boldsymbol{K}(v)$ are plotted for $v=1,2, \ldots, 20$ which fall into a plane in $\boldsymbol{K}$-space perpendicular to a 5 -fold axis. Besides the individual points one notes point clusters on lines which form pentagonal shapes. From the construction, the individual points appear on lines at distances of the form $(n+m \Phi)$ where

$$
\Phi=\frac{1}{2}(1+\sqrt{5}), \quad n, m=0, \pm 1, \pm 2, \ldots .
$$

The clusters appear at the values

$$
(n, m)=\left(f_{\mu+1}, 0\right) ;\left(0, f_{\mu}\right),
$$

where $f_{\mu}$ are the Fibonacci numbers,

$f_{0}=f_{1}=1, \quad f_{\mu+1}=f_{\mu}+f_{\mu-1}, \quad \mu=1,2, \ldots$.

By the interference in (5.24), these clusters will give marked contributions to the diffraction pattern. Figure 3 does not yet show the interference of the 


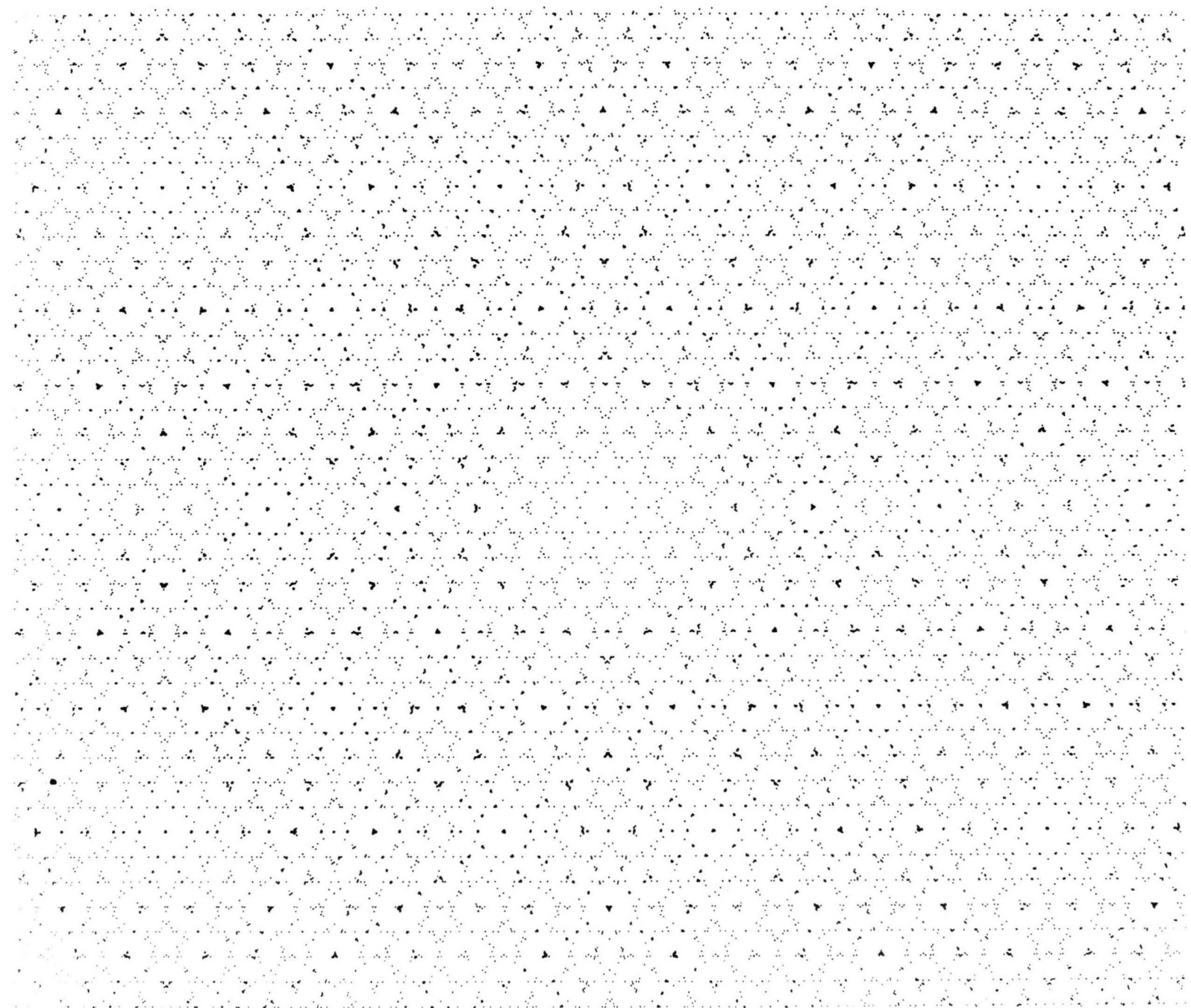

Fig. 3. Points in $\boldsymbol{K}$-space in a plane orthogonal to a 5 -fold axis through the origin in the long-range approximation. Each point marks a diffraction spot. With increasing distance from the center, point cluster appear at positions determined by the Fibonacci numbers.

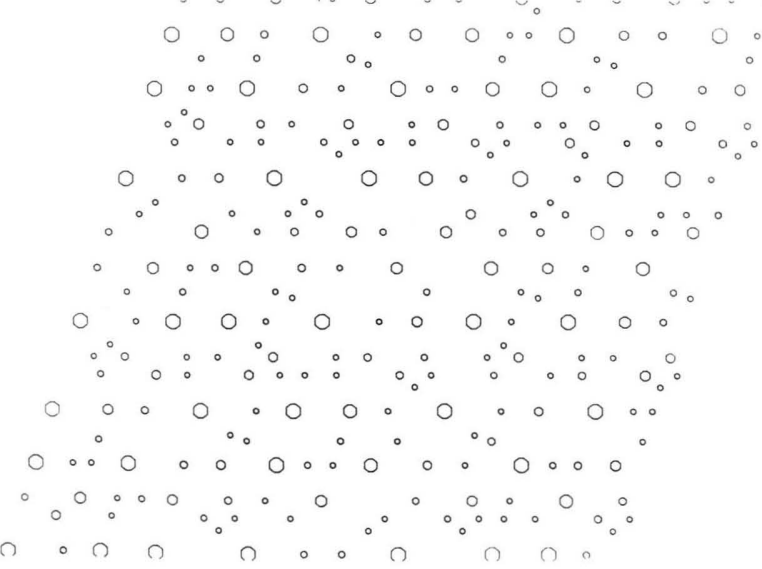

Fig. 4. Diffraction pattern in $\boldsymbol{K}$-space with the same geometry as Fig. 3, computed in the long-range approximation. The sharp spots of Fig. 3 have been replaced by distributions, and the interference according to (5.24) has been taken into account. The area of the circles is a measure of the intensity. The strongest intensities appear at the positions of the Fibonacci clusters. These positions form a pentagon pattern. Shown is a sector $2 \pi / 5$ with the center of $\boldsymbol{K}$-space at the left bottom corner. 
individual contributions in (5.24). To get interference, one should replace the $\delta$-functions in this equation by reasonable distributions in $\boldsymbol{K}$-space. In Fig. 4 the resulting pattern is shown. The Fibonacci clusters clearly dominate through the interference effects, and the pattern resembles closely the experimental results given in [1] and [2,3].

The long-range approximation is a first step in the analysis of the diffraction pattern. To improve it one could start from an analysis of the neglected part $\boldsymbol{v}_{b}$ given in (5.7).

\section{The Shift Parameters and the Point Symmetry of the Quasilattice}

In the present section, we explore the range of values of the shift parameters $\gamma_{i}$ and the possible point symmetry of the hexagrid and quasilattice. The shift parameters have a natural interpretation as a vector in $\mathbb{E}^{6}$, compare Section 2. Since in $\mathbb{E}^{6}$ there is a hexagrid $Y$ with translational order, the vector $\gamma$ can be chosen from the first hypercell which serves as a fundamental region. This choice leads to (2.2) and (3.1). Within the first hypercell, one should now find a fundamental region $\Gamma$ with respect to the action of the hyperoctahedral point group $\Omega(6)$. A natural choice for this fundamental region would be

$$
\Gamma=\left\{\gamma \mid \gamma_{1} \geqq \gamma_{2} \geqq \gamma_{3} \geqq \gamma_{4} \geqq \gamma_{5} \geqq \gamma_{6} \geqq 0\right\} .
$$

Within this fundamental region, one should explore the stability groups for the points $\gamma$ under the action of the full space group in $\mathbb{E}^{6}$.

Turning now to the projected hexagrid $Y_{1}$ in $\mathbb{E}^{3}$, the range and significance of the shift parameters $\gamma_{i}$ should be explored with respect to a classification of all possible different hexagrids. First of all one observes that upon projecting the vector $\gamma$ as

$$
\gamma=\gamma_{1}+\gamma_{2}
$$

into the subspaces $\mathbb{E}_{1}^{3}$ and $\mathbb{E}_{2}^{3}$, a shift in $\gamma_{1}$ yields a rigid translation of the hexagrid $Y_{1}$ and does not change its intrinsic structure. Secondly it is clear that the values of $\gamma_{2}$ should be classified according to the action of the icosahedral group $\mathrm{A}(5)$. A natural choice for the analysis seems to be then that $\gamma$ is chosen from a fundamental region in $\Gamma$ with respect to $\mathbb{E}^{6}$ and that in addition one requires

$$
\gamma_{1}=0 \text {. }
$$

This choice will now be explored, but we mention already here that it does not exhaust the full classification. With the choice (6.3), the shift parameters $\gamma_{i}$ are given by

$$
\begin{aligned}
& \gamma_{i}=\left(\gamma_{2} \cdot \boldsymbol{e}_{i 2}\right), \\
& -\frac{1}{2}<\left(\boldsymbol{\psi}_{2} \cdot \boldsymbol{e}_{i 2}\right) \leqq \frac{1}{2}, \quad i=1,2, \ldots, 6 .
\end{aligned}
$$

Since the vectors $\boldsymbol{e}_{i_{2}}, i=1,2, \ldots, 6$, point to the centers of six faces of a dodecahedron in $\mathbb{E}_{2}^{3}$, the vector $\gamma_{2}$ is restricted to the interior of a dodecahedron whose faces have the distance $1 / 2$ from the origin.

With this restriction, we look for a fundamental region with respect to the action of the group A (5). As shown in Sect. 3, the points of the space $\mathbb{E}_{2}^{3}$ under $\mathrm{A}(5)$ are transformed by the representation $D^{\omega}$. It was shown in (2.14) that this representation, by use of the automorphism $\sigma$, is related to the representation $D^{i}$. The representation $D^{i}$ consists of the rigid proper rotations which transform a dodecahedron in $\mathbb{E}^{3}$ into itself. A choice of a fundamental region $\Gamma^{2}$ for this action in $\mathbb{E}^{3}$ is shown in Figure 5. It is defined by

$$
\begin{aligned}
\Gamma^{2}= & \left\{\gamma_{2} \mid \gamma_{2}=\lambda_{1} e_{12}+\lambda_{4} e_{42}-\lambda_{6} e_{62}, \lambda_{1} \geqq \lambda_{4}, \lambda_{6} \geqq \lambda_{4},\right. \\
& \text { if } \lambda_{1}=\lambda_{4} \neq 0 \text { then } \lambda_{6}=\lambda_{1}, \\
& \text { if } \lambda_{6}=\lambda_{4} \neq 0 \quad \text { then } \lambda_{1} \geqq \lambda_{4}, \\
& \text { if } \left.\lambda_{4}=0 \quad \text { then } \lambda_{6} \geqq \lambda_{1}\right\} .
\end{aligned}
$$

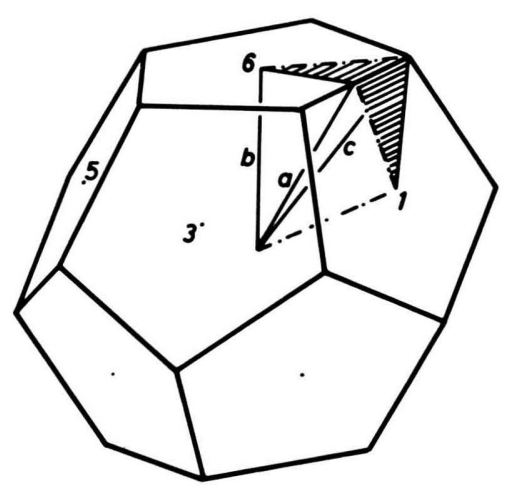

Fig. 5. The fundamental region $\Gamma^{2}$ for the vector $\gamma_{2}$ in $\mathbb{E}_{2}^{3}$. The faces of the dodecahedron are marked according to the primed numbers of Figure 1 . The shaded part of the faces determines the directions of $\gamma_{2}$ in the fundamental region. The particular lines marked $a, c$ and $b$ yield the point symmetry groups $\mathrm{C}(2), \mathrm{C}(3)$ and $\mathrm{C}(5)$ of the hexagrid and quasilattice while the origin of the dodecahedron yields the point symmetry group $\mathrm{A}(5)$. 
Table 7. Point symmetry of the hexagrid $Y_{1}$ and quasilattice $Z_{1}$ for special values of the coefficients $\lambda_{1}, \lambda_{4}, \lambda_{6}$ in the expression for $\gamma_{2}$ of (6.6). The letters $a, b, c$ refer to Figure 5 .

\begin{tabular}{lll}
\hline Point & $\begin{array}{l}\text { Point } \\
\text { in } \mathbb{E}_{2}^{3}\end{array}$ & Value of $\lambda_{1}, \lambda_{4}, \lambda_{6}$ \\
\hline $\mathrm{C}(2)$ & $a$ & $\lambda_{1}=\lambda_{6}, \lambda_{4}=0,0<\lambda_{1} \leqq(1+\theta)^{-1 / 2} / 2$ \\
$\mathrm{C}(3)$ & $c$ & $\lambda_{1}=\lambda_{4}=\lambda_{6}, 0<\lambda_{1} \leqq(1+2 \theta)^{-1 / 2} / 2$ \\
$\mathrm{C}(5)$ & $b$ & $\lambda_{1}=\lambda_{4}=0,0<\lambda_{6} \leqq 1 / 2$ \\
$\mathrm{~A}(5)$ & & $\lambda_{1}=\lambda_{4}=\lambda_{6}=0$ \\
\hline
\end{tabular}

Table 8. Point symmetry of the hexagrid $Y_{1}$ and quasilattice $Z_{1}$ for values of the parameters $\gamma_{i}$ which correspond to points on the boundary of the first hypercell in $\mathbb{E}^{6}$. The space group symmetry of these point in $\mathbb{E}^{6}$ induces a point symmetry in $\mathbb{E}^{3}$.

\begin{tabular}{lllllllll}
\hline $\begin{array}{l}\text { Point } \\
\text { group } \\
\text { in } \mathbb{E}^{3}\end{array}$ & $\begin{array}{l}\text { Gen- } \\
\text { erators }\end{array}$ & $\gamma_{1}$ & $\gamma_{2}$ & $\gamma_{3}$ & $\gamma_{4}$ & $\gamma_{5}$ & $\gamma_{6}$ & range \\
\hline $\mathrm{C}(2)$ & $g_{2}$ & $\frac{1}{2}$ & $\gamma_{2}$ & $\frac{1}{2}$ & 0 & 0 & $\gamma_{2}$ & $-\frac{1}{2}<\gamma_{2} \leqq \frac{1}{2}$ \\
& & $\gamma_{1}$ & $\frac{1}{2}$ & $\gamma_{1}$ & 0 & 0 & $\frac{1}{2}$ & $-\frac{1}{2}<\gamma_{1}<\frac{1}{2}$ \\
$\mathrm{C}(3)$ & $g_{3}$ & $\frac{1}{2}$ & $\frac{1}{2}$ & $\gamma_{3}$ & $-\gamma_{3}$ & $\gamma_{3}$ & $\frac{1}{2}$ & $-\frac{1}{2}<\gamma_{3} \leqq \frac{1}{2}$ \\
& & $\gamma_{1}$ & $\gamma_{1}$ & $\frac{1}{2}$ & $-\frac{1}{2}$ & $\frac{1}{2}$ & $\gamma_{1}$ & $-\frac{1}{2}<\gamma_{1}<\frac{1}{2}$ \\
$\mathrm{C}(5)$ & $g_{5}$ & $\frac{1}{2}$ & $\frac{1}{2}$ & $\frac{1}{2}$ & $\frac{1}{2}$ & $\frac{1}{2}$ & $\gamma_{6}$ & $-\frac{1}{2}<\gamma_{6}<\frac{1}{2}$ \\
& & $\gamma_{1}$ & $\gamma_{1}$ & $\gamma_{1}$ & $\gamma_{1}$ & $\gamma_{1}$ & $\frac{1}{2}$ & $-\frac{1}{2}<\gamma_{1}<\frac{1}{2}$ \\
$\mathrm{~A}(5)$ & $g_{2}, g_{5}$ & $\frac{1}{2}$ & $\frac{1}{2}$ & $\frac{1}{2}$ & $\frac{1}{2}$ & $\frac{1}{2}$ & $\frac{1}{2}$ & \\
& & & & & & &
\end{tabular}

In the last three lines of (6.6) the boundaries of $\Gamma^{2}$ are specified. The fundamental region $\Gamma^{2}$ of $\mathbb{E}_{2}^{3}$ contains four special types of points with stability groups $\mathrm{C}(2), \mathrm{C}(3), \mathrm{C}(5)$ and $\mathrm{A}(5)$ respectively. Under a general element $g \in \mathrm{A}(5)$, the shift parameters according to $(2.9,2.18)$ transform as

$$
g: \gamma_{i} \rightarrow \bar{\gamma}_{i}=\sum_{j=1}^{6} \gamma_{j} \tilde{D}_{j i}^{6}(g) .
$$

This transformation yields in $\mathbb{E}_{1}^{3}$ a map from a hexagrid $Y_{1}$ to a new hexagrid $\bar{Y}_{1}$. If now the element $g$ is chosen from the stability group of a particular vector

$$
\gamma=\gamma_{2},
$$

this vector $\gamma$ is stable under $g$ and hence the hexagrids $Y_{1}$ and $\bar{Y}_{1}$ coincide. In other words, the stability groups of $\gamma_{2}$ in $\mathbb{E}_{2}^{3}$ yield point symmetry groups of the hexagrid $Y_{1}$ in $\mathbb{E}_{1}^{3}$. If the hexagrid has a point symmetry, the process of dualization shows that the dual quasilattice $Z_{1}$ must have the same point symmetry.

The present analysis exhausts all cases where the shift vector $\gamma$ fulfil the conditions Eq. (6.3). If $\gamma_{2}$ is varied within the fundamental region $\Gamma^{2}$, one obtains hexagrids $Y_{1}$ and $Z_{1}$ which are inequivalent under the group A (5), except in the cases of Table 7 in which they have a point symmetry. If $\gamma_{2}$ is taken from a region outside $\Gamma^{2}$ but inside the dodecahedron determined by (6.5), the corresponding hexagrid $Y_{1}$ and $Z_{1}$ can be related by a rigid rotation, corresponding to an element of $\mathrm{A}(5)$, to a hexagrid constructed from a point of $\Gamma^{2}$.

We turn now to the action of the group A (5) on the full space $\mathbb{E}^{6}$. This action is represented in the subspaces $\mathbb{E}_{1}^{3}$ and $\mathbb{E}_{2}^{3}$ by the simultaneous transformation with $D^{i}(g)$ and $D^{\omega}(g)$. A fundamental region for $\mathbb{E}_{2}^{3}$ is determined by $\Gamma^{2}$ given in (6.6). Because of the automorphism $\sigma$ constructed in (2.14), there is a fundamental region corresponding to $\Gamma^{2}$ in $\mathbb{E}_{1}^{3}$. The stability groups of corresponding points in $\mathbb{E}_{1}^{3}$ and $\mathbb{E}_{2}^{3}$ are linked by the automorphism $H \rightarrow \sigma(H)$. But then one can use a translational shift in $\mathbb{E}_{1}^{3}$ to put $\gamma_{1} \rightarrow 0$ and recover the conditions on $\Gamma^{2}$ analyzed before.

It is important to note that this analysis fails in certain regions of the first hypercell in $\mathbb{E}^{6}$. The reason is that, on the boundary of this hypercell, there are points which are stable under elements from the full space group of the hexagrid in $\mathbb{E}^{6}$. For

$$
\gamma_{i}=\sqrt{2}\left(\boldsymbol{\gamma} \cdot \boldsymbol{c}_{i}\right)=\frac{1}{2}, \text { fixed } i,
$$

the point $\gamma$ is on a hyperplane from the hexagrid which is a hyperreflection plane of the hexagrid generated from the space group. If $\gamma$ sits on the intersection of several such hyperplanes, these hyperreflections generate a point symmetry group at a point in $\mathbb{E}^{6}$ on the boundary.

If moreover this point symmetry group is a subgroup of $\mathrm{A}(5)$, one obtains values of the shift parameters $\gamma_{i}$ which yield a hexagrid $Y_{1}$ with a point symmetry. Since we are dealing with a stability group formed from space group elements in $\mathbb{E}^{6}$, the vector $\gamma$ itself does not show this point symmetry, contrary to the case discussed before. In Table 8 we give values of the parameters $\gamma_{i}$ which correspond to hexagrids $Y_{1}$ and quasilattices $Z_{1}$ with this type of point symmetry.

\section{Composite Cells and Local Symmetry Breaking}

In the construction of the quasilattice $Z_{1}$ from the hexagrid $Y_{1}$ described in Sects. 3 and 4, we assumed 
that the hexagrid $Y_{1}$ be regular, i.e. that at most three planes of $Y_{1}$ intersect in a vertex. We now relax this condition and study local vertices of $Y_{1}$ where $p, 3 \leqq p \leqq 6$, planes intersect. We form a star from the $p$ vectors $\left\{ \pm \boldsymbol{e}_{\alpha 1}\right\}$ which belong to these intersecting planes, and we call two stars inequivalent if they cannot be transformed into one another by a transformation from the icosahedral group $\mathrm{A}(5)$.

It is easily found that there are five inequivalent stars, two for $p=3$, and one for $p=4,5,6$ respectively. It follows that the hexagrid $Y_{1}$ may have five inequivalent types of vertices. Consider now the quasilattice $Z_{1}$ dual to $Y_{1}$. For each vertex of $Y_{1}$ one obtains a cell of $Z_{1}$. The cells must have rhombus faces since these faces are dual to edges of $Y_{1}$ formed from pairs of planes, compare Section 4 . The local result of the dualization leads to the family of five zonohedra known from the theory of polyhedra, compare Coxeter [9]. These zonohedra are described in Table 9 and shown in Figure 2. According to Coxeter [9], the rhombic triakontahedron was found by Kepler, (1619), the rhombic icosahedron by Fedorov (1885) and the rhombic dodecahedron by Bilinski (1960). The new result for the quasilattice can be stated as follows: The infinite space filling or quasilattice $Z_{1}$ of $\mathbb{E}^{3}$ dual to a general hexagrid $Y_{1}$ consists of convex cells which are the five zonohedra described in Table 9.

Now we consider changes in the parameters $\gamma_{i}$ of the hexagrid $Y_{1}$ which induce changes in the cell structure of $Z_{1}$. We start with a vertex of $Y_{1}$ where $p, 3<p \leqq 6$ planes intersect. If one of these planes is removed from the vertex by a small change of the corresponding shift parameter, the vertex remains as the intersection of $p-1$ planes. Close to this vertex there appear $(p-1)(p-2) / 2$ new vertices formed from the intersection of the removed plane with pairs of remaining planes. In the dual quasi-

Table 9. The five zonohedra which appear in the infinite quasilattice $Z_{1}$ as cells dual to the intersection of $p$ planes in the hexagrid $Y_{1}$. Compare Figure 2.

\begin{tabular}{llll}
\hline$p$ & Symbol & Name & $\begin{array}{l}\text { Local point } \\
\text { group }\end{array}$ \\
\hline 3 & $h$ & rhombic hexahedron, thick & $\mathrm{D}_{3}$ \\
3 & $h^{\prime}$ & rhombic hexahedron, thin & $\mathrm{D}_{3}$ \\
4 & $d$ & rhombic dodecahedron & $\mathrm{D}_{2}$ \\
5 & $i$ & rhombic icosahedron & $\mathrm{D}_{5}$ \\
6 & $t$ & rhombic triakontahedron & $\mathrm{A}(5)$ \\
\hline
\end{tabular}

Table 10. Decomposition of the composite cells into smaller composite cells and into elementary cells.

\begin{tabular}{llc}
\hline Cell & Composite content & Elementary content \\
\hline$d$ & $d$ & $2 h+2 h^{\prime}$ \\
$i$ & $d+\left(3 h+3 h^{\prime}\right)$ & $5 h+5 h^{\prime}$ \\
$t$ & $i+\left(5 h+5 h^{\prime}\right)$ & $10 h+10 h^{\prime}$ \\
\hline
\end{tabular}

Table 11. The symmetry group $\mathrm{H}$ of the composite cell, the stability group $\mathrm{K}$ of the filled composite cell, and the number $x$ of different packings for the composite cell.

\begin{tabular}{llll}
\hline Cell & $\mathrm{H}$ & $\mathrm{K}$ & $x$ \\
\hline$d$ & $\mathrm{D}_{2}$ & $\mathrm{C}(2)$ & 2 \\
$i$ & $\mathrm{D}_{5}$ & $\mathrm{C}(2)$ & 5 \\
$t$ & $\mathrm{~A}(5)$ & $\mathrm{e}$ & 60 \\
\hline
\end{tabular}

lattice $Z_{1}$ we start with a local zonohedron for $p>3$. Upon removal of the plane, this zonohedron is decomposed into a zonohedron corresponding to $p-1$ and into a zone formed from $(p-1)(p-2) / 2$ rhombic hexahedra. These hexahedra are packed and have each four edges orthogonal to the removed plane. This process of removal can be repeated and leads to a complete decomposition of the zonohedra $t, i$ and $d$ into the elementary zonohedra $h$ and $h^{\prime}$. The numbers of the pieces are given in Table 10. The full decomposition of the triakontahedron was given by Kowalewski [10] in 1938.

We call the cells $t, i$ and $d$ composite and the cells $h$ and $h^{\prime}$ elementary. The symmetry groups $K$ for these polyhedral cells are given in Table 10. Consider now a composite cell and its filling by elementary cells. By $H$ we denote the subgroup of $K$ which transforms both the composite cell and its filling into itself while interchanging and rotating the elementary cells. All operations of $\mathrm{K}$ not in $\mathrm{H}$ lead to different fillings of the composite cell. The numer $x$ of different fillings are listed in Table 11.

Consider now a continuous change of one of the shift parameters with respect to a vertex formed from $p-1$ planes. When the corresponding plane passes through the vertex, the composite cell must jump from an initial filling by elementary cells to a new filling. It is of interest to consider the physics of these jumps which imply a local discontinuous rearrangement of the elementary cells. It corresponds to a discontinuous transition between two configurations where the symmetry group $\mathrm{H}$ of the composite cells is broken. 
Having obtained the form of the composite cells and their local point symmetry, one could ask about the possibility of global point symmetries. This question can be answered through the analysis given in Section 6. The cases described in Table 8 yield quasilattices $Z_{1}$ with a global point symmetry and with a central composite cell that has the same local point symmetry. In the last case of Table 8 one obtains a quasilattice $Z_{1}$ with a triakontahedron at the center and full icosahedral point symmetry.

\section{Two-Dimensional Layers of Cells in the Quasilattice}

We analyze a fixed plane of the hexagrid $Y_{1}$ in $\mathbb{E}^{3}$ whose points $\boldsymbol{x}$ obey

$$
\boldsymbol{x} \cdot \boldsymbol{e}_{61}=\frac{1}{2} k_{6}-\gamma_{6} .
$$

Consider the layer of cells of $Y_{1}$ which have one face common with this plane and whose points are on the side of this plane determined by

$$
\frac{1}{2}\left(k_{6}-2\right)<\boldsymbol{y} \cdot \boldsymbol{e}_{61}+\gamma_{6} \leqq \frac{1}{2} k_{6} .
$$

The index system for any such cell has the value $\left\{k_{1}(\boldsymbol{y}), k_{2}(\boldsymbol{y}), \ldots, k_{6}(\boldsymbol{y})\right\}$ with

$$
\begin{aligned}
& \left.k_{j}(\boldsymbol{y})=2-\left(\left(\boldsymbol{y} \cdot \boldsymbol{e}_{j 1}\right)+\gamma_{j}-\frac{1}{2}\right)\right\rceil+1, \\
& j=1,2, \ldots, 5, \quad k_{6}(\boldsymbol{y})=k_{6} .
\end{aligned}
$$

Now we define the index function $J(y)$ by

$$
\begin{aligned}
J(\boldsymbol{y}): & =\frac{1}{2} \sum_{\alpha=1}^{6} k_{\alpha} \\
& =\frac{1}{2}\left(k_{6}+5\right)+\sum_{j=1}^{5}\left(\left\lceil\left(\left(\boldsymbol{y} \cdot \boldsymbol{e}_{j_{1}}\right)+\gamma_{i}-\frac{1}{2}\right)\right\rceil\right)
\end{aligned}
$$

and the related function

$\left.J_{0}(\boldsymbol{y})=\frac{1}{2}\left(k_{6}+5\right)+-\sum_{j=1}^{5}\left(\left(\boldsymbol{y} \cdot \boldsymbol{e}_{j 1}\right)+\gamma_{j}-\frac{1}{2}\right)\right\rceil$.

Both functions can be evaluated on the face incident with the plane specified in (8.1). By writing for the points of this plane

$$
\boldsymbol{x}=\left(\frac{1}{2} k_{6}-\gamma_{6}\right) \boldsymbol{e}_{61}+\boldsymbol{z}
$$

one finds that $z$ is a vector in this plane. From the direction of the vectors $\boldsymbol{e}_{11}, \boldsymbol{e}_{21}, \ldots, \boldsymbol{e}_{51}$ one easily gets

$$
\sum_{j=1}^{5} \boldsymbol{x} \cdot \boldsymbol{e}_{j 1}=\sqrt{5}\left(\frac{1}{2} k_{6}-\gamma_{6}\right)=q\left(k_{6}, \gamma_{6}\right) .
$$

Hence the function $J_{0}(\boldsymbol{y})$ becomes

$$
\begin{aligned}
J_{0}(\boldsymbol{y}) & =J_{0}(\boldsymbol{x}) \\
& =\frac{1}{2}\left(k_{6}+5\right)+\left\lceil\left(q\left(k_{6}, \gamma_{6}\right)+\sum_{j}^{5}\left(\gamma_{j}-\frac{1}{2}\right)\right)\right\rceil
\end{aligned}
$$

independent of the position on the plane. Now we note that the function $\left.{ }^{-}\right\rceil$has the property

$$
\left.\left.0 \leqq \sum_{i=1}^{n}-\alpha_{i}\right\rceil--\sum_{i=1}^{n} \alpha_{i}\right\rceil \leqq n-1 .
$$

The application of this inequality to the index sum function $J(y)$ yields

$$
0 \leqq J(y)-J_{0}(y) \leqq 4 .
$$

It follows that $J(y)$ for the layer of cells associated with the plane can take the value

$$
J(\boldsymbol{y})=J_{0}+m, \quad m=0,1,2,3,4 .
$$

The value $J_{0}$ depends only on chosen plane, not on the position of the cell face within that plane.

For the second layer of cells whose points obey

$$
\frac{1}{2} k_{6}<\boldsymbol{y} \cdot \boldsymbol{e}_{61}+\gamma_{6} \leqq \frac{1}{2}\left(k_{6}+1\right)
$$

the index system differs from the one of their neighbours on the first side of the plane in the single index $k_{6}^{\prime}=k_{6}+1$. It follows that their index sum function takes the values

$$
J(\boldsymbol{y})=J_{0}+m^{\prime}, \quad m^{\prime}=1,2,3,4,5 .
$$

The analysis can be repeated for planes of the hexagrid associated with other faces of the dodecahedron. Suppose the vector $\boldsymbol{e}_{j 1}$ is chosen to define the corresponding plane. Before defining the index system one should now replace the remaining vectors $\boldsymbol{e}_{i 1}, i \neq j$ by a set $\tilde{\boldsymbol{e}}_{i 1}, i \neq j$ where $\tilde{\boldsymbol{e}}_{i 1}= \pm \boldsymbol{e}_{i 1}$ is chosen in such a way that

$$
\tilde{\boldsymbol{e}}_{i 1} \cdot \boldsymbol{e}_{j 1}=\theta, \quad i \neq j, \quad i=1,2, \ldots, 6 .
$$

Now we analyze the part of the quasilattice $Z_{1}$ dual to the two layers of the hexagrid associated with the plane (8.1). The faces of a cell from the first layer and incident with the plane (8.1) form polygons. At any vertex of this polygon there must start an edge of the cell which is not in the plane and is common to four cells from the first layer. Upon dualization of the hexagrid, the four vertices of $Z_{1}$ dual to these four cells form the vertices of a rhombus face. The first layer of $Y_{1}$ then must have as its dual a rhombus net whose vertices are formed from the vectors $\boldsymbol{e}_{11}, \boldsymbol{e}_{21}, \ldots, \boldsymbol{e}_{51}$. Similarly, the second layer 


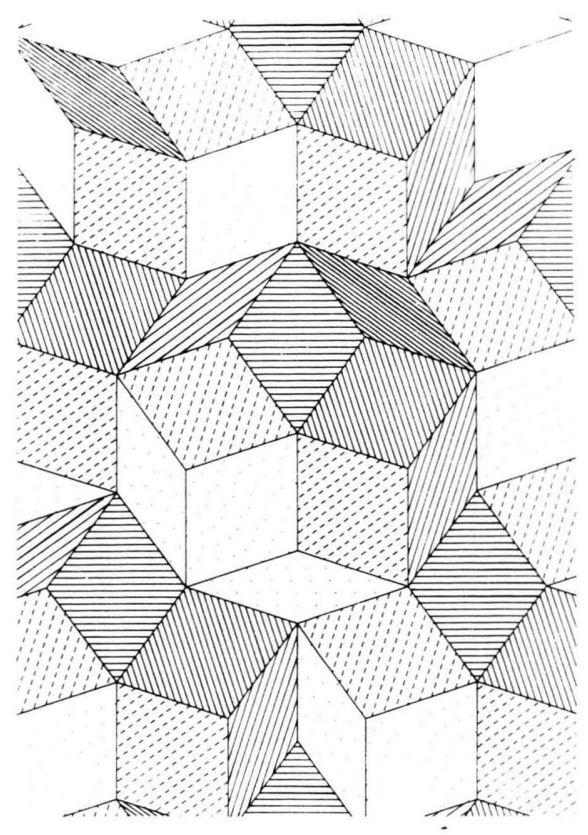

Fig. 6. A layer of packed cells in the quasilattice. All rhombic cells have 4 edges orthogonal to the plane of this figure and are packed with respect to the corresponding faces. In this projection, the other rhombus faces appear in the form of two types of rhombus shapes known from the Penrose pattern. The surface in $\mathbb{E}^{3}$ formed by the top faces of the layer is indicated by a hatching.

of $Y_{1}$ associated with the plane has as its dual the same rhombus net shifted in the dual space by the vector $\boldsymbol{e}_{61}$ in view of (8.11) and (8.13). The vertices of the two nets are linked by the edges formed from the vector $\boldsymbol{e}_{61}$.

The two layers of cells of $Y_{1}$ associated with the fixed plane then have as their dual a single layer of rhombohedral cells from $Z_{1}$. The cells of this dual layer have each four edges formed by the vector $\boldsymbol{e}_{61}$ and four faces whose normal vector is orthogonal to $\boldsymbol{e}_{61}$. The other faces of all these cells form two parallel rhombus nets whose vertices are connected in pairs by the vector $\boldsymbol{e}_{61}$.

In Fig. 6 we give a projection of this layer seen in the direction opposite to the vector $\boldsymbol{e}_{61}$. The inclination of the upper rhombus net is indicated by a hatching.

At each vertex of this layer of $Z_{1}$ one could mark the sum index function $J$ of (8.4). From the condi- tions (8.13) it follows that, in a rhombus net, the number $J$ takes at most five values. The projection of a rhombus onto the plane yields two types of rhombus shapes, depending on the orientation of the single original type of rhombus. These two projected types of rhombus coincide with the ones appearing in the rhombus form of the plane Penrose pattern. De Bruijn [5] has shown that the Penrose patterns are restricted by an additional condition which admits for a corresponding index sum function only four values $1,2,3,4$. It follows that the projection of the present three-dimensional rhombus net onto a plane in general does not yield a Penrose pattern.

\section{Conclusion}

In [4] a general method was introduced for the construction of space fillings and implemented in particular for the concept of a hexagrid and space filling of $\mathbb{E}^{3}$ associated with the icosahedral group. In the present paper the geometric analysis and classification announced in [4] is given. It is believed that this analysis gives insight into the structure of this quasilattice and may stimulate a similar analysis for others.

As an example of a global property, the structure factor is treated in Section 5. In a recent analysis of the stability of the quasilattice in terms of the Landau theory, Bak [11] employed the projection from $\mathbb{E}^{6}$ which was introduced by us in [4]. We believe that the classification theory presented here should be important for the stability problem. Similarly it should have its bearing on the structure and dynamics of electron and phonon states in a quasilattice. With respect to the local properties examined in Sects. 6-8, we expect that experimental evidence on it will be available in the near future.

\section{Acknowledgement}

The author would like to thank L. Kramer for the computation and graphic representation given in Figs. 3, 4 and 6. 
[1] D. Shechtman, I. Blech, D. Gratias, and J. W. Cahn, Phys. Rev. Lett. 53, 1951.(1984).

[2] P. A. Bancel, P. A. Heiney, P. W. Stephens, A. I. Goldman, and P. M. Horn, preprint, April 1985.

[3] D. R. Nelson and B. I. Halperin, preprint, April 1985.

[4] P. Kramer and R. Neri, Acta cryst. A40, 580 (1984).

[5] N. G. de Bruijn, Indagationes Mathematicae 43, 39, 53 (1981).

[6] H. S. M. Coxeter and W. O. Moser, Generators and Relations for Discrete Groups, Springer-Verlag, Berlin 1965 .
[7] A. L. Mackay, Sov. Phys. Crystallography 26, 517 (1981); Physica 114 A, 609 (1982).

[8] M. Lax, Symmetry Principles in Solid State and Molecular Physics, Wiley, New York 1974.

[9] H. S. M. Coxeter, Regular Polytopes, MacMillan, New York 1963.

[10] G. Kowalewski, Der Keplersche Körper und andere Bauspiele, K. F. Koehlers Antiquarium, Leipzig 1938.

[11] P. Bak, Phys. Rev. Lett. 54, 1517 (1985). 